\title{
CELL SEGMENTATION VIA SPECTRAL ANALYSIS ON PHASE RETARDATION FEATURES
}

\author{
Hang Su $\quad$ Zhaozheng Yin ${ }^{\ddagger} \quad$ Seungil Huh $\quad$ Takeo Kanade $^{\dagger}$ \\ * Department of Electronic Engineering, Shanghai Jiaotong University \\ $\dagger$ The Robotics Institute, Carnegie Mellon University \\ $\ddagger$ Department of Computer Science, Missouri University of Science and Technology
}

\begin{abstract}
In this paper, the authors propose a cell segmentation algorithm via spectral analysis over phase retardation features, which are derived from the optical principle of phase contrast microscopy image formation process. Images are first partitioned into phase-homogeneous atoms by clustering neighboring pixels with similar phase retardation features. Cell segmentation is then performed by clustering the atoms into several clusters using multi-class spectral analysis. Experimental results demonstrate that our method generates quality cell segmentation results and outperforms previous methods.
\end{abstract}

Index Terms - phase contrast microscopy image analysis, phase retardation feature, spectral analysis, cell segmentation

\section{INTRODUCTION}

Cell segmentation in phase contrast microscopy images is one of the most fundamental and important problems in computeraided cell image analysis. This task is challenging due to low contrast between foreground and background in microscopy images, indistinct boundaries among cells, and the artifacts caused by phase contrast optics such as bright halos and shade-off.

It is known that the imaging mechanism of phase contrast microscopy, which employs interference of optics, is completely different from the general imaging system for natural images [1]. Despite this fact, the majority of cell segmentation approaches, such as thresholding, watershed method [2] and level sets [3], treat microscopy images as general natural images. In contrast, in our recent work [4], we proposed a way to represent a phase contrast microscopy image as a linear combination of diffraction patterns based on the phase contrast image model introduced by Yin et al. [5]. In the method, each pixel is represented by a phase retardation feature vector, with which cell detection can be effectively performed [4].

In this paper, we further explore the phase retardation features to achieve quality cell segmentation. First, a

This work is supported by funds from Cell Image Analysis Consortium of Carnegie Mellon University and University of Missouri Research Board. phase contrast microscopy image is partitioned into phasehomogeneous atoms by clustering neighboring pixels with similar phase retardation features. The distribution of phase retardation features within an atom is then modeled with a multivariate Gaussian model, and the similarities between an atom and its neighboring atoms are measured using JensenShannon divergence [6], resulting in an affinity matrix among the atoms. Cell segmentation is subsequently performed using a multi-class spectral clustering technique [7] over the matrix with the number of clusters automatically determined by rate distortion theory [6].

Our experiments show that the proposed algorithm can segment individual cells out of background with a high quality and outperforms previous methods. Since our method separates cells based on phase retardation, the segmentation results are also useful for cell stage classification (e.g., intermitosis vs. mitosis/apoptosis).

\section{METHODOLOGY}

In [4], we derived a dictionary-based imaging model for phase contrast optics as

$$
\mathbf{g} \approx \sum_{k=1}^{K} \psi_{k}(\mathbf{x}) \operatorname{PSF}\left(\theta_{k}\right),
$$

where $\mathbf{g}$ is the intensity of an observed image, $K$ is the number of representative phase retardations, and $\operatorname{PSF}\left(\theta_{k}\right)$ denotes the basis with phase retardation $\theta_{k}$ from a dictionary. In this model, each pixel at position $\mathbf{x}=\left(x^{r}, x^{c}\right)$ is represented by a feature vector $\boldsymbol{\Psi}(\mathbf{x})=\left[\psi_{1}(\mathbf{x}), \cdots, \psi_{k}(\mathbf{x}), \cdots, \psi_{K}(\mathbf{x})\right]^{T}$, where $\psi_{k}(\mathbf{x}) \in[0,1]$.

In this section, we first present how to partition a phase contrast microscopy image into phase-homogeneous atoms by clustering phase retardation feature vectors of pixels. We then apply the multi-class spectral analysis technique [7] to segment cells by clustering the phase-homogeneous atoms.

\subsection{Atom generation based on phase retardation features}

We advocate a method to partition a microscopy image into atoms and utilize them as elementary units for cell segmentation rather than using pixels as units. Atom partitioning eliminates local redundancy and avoids over-segmented noisy clips. It also allows to incorporate local information (e.g., 
phase retardation distribution in an atom) rather than using pixelwise information (e.g., intensity of a pixel).

Atoms are generated by clustering neighboring pixels based on pairwise similarity between phase retardation features of pixels. (Note that conventional superpixel extraction is conducted based on image intensity or color values of pixels.) As a result, the phase retardations within an atom is statistically homogeneous, thus we call the atom phasehomogeneous atom. More formally, given two feature vectors at position $\mathbf{x}_{i}$ and $\mathbf{x}_{j}$, the dissimilarity between $\boldsymbol{\Psi}\left(\mathbf{x}_{i}\right)$ and $\boldsymbol{\Psi}\left(\mathbf{x}_{j}\right)$ is defined as

$$
d_{p}=\left\|\boldsymbol{\Psi}\left(\mathbf{x}_{i}\right)-\mathbf{\Psi}\left(\mathbf{x}_{j}\right)\right\|
$$

which is upper bounded by

$$
d_{p} \leqslant\left\|\boldsymbol{\Psi}\left(\mathbf{x}_{i}\right)\right\|+\left\|\boldsymbol{\Psi}\left(\mathbf{x}_{j}\right)\right\| \leqslant 2 \sqrt{K} .
$$

And the spatial distance between $\mathbf{x}_{i}$ and $\mathbf{x}_{j}$ is measure by

$$
d_{s}=\left\|\mathbf{x}_{i}-\mathbf{x}_{j}\right\|
$$

On the assumption that all atoms have the same size, the distance between two pixels belonging to the same atom is upper bounded by the atom size $S=P / N$, where $P$ is the number of pixels in the image and $N$ is a user-specified number of atoms.

The partition of atoms $\left\{\mathcal{A}_{n}\right\}_{n=1}^{N}$ is then determined by minimizing the normalized distortion of phase retardation features and spatial positions as

$$
\min \sum_{n=1}^{N} \sum_{\mathbf{x} \in \mathcal{A}_{n}}\left(\frac{1}{4 K}\left\|\mathbf{\Psi}(\mathbf{x})-\boldsymbol{\mu}_{p}^{(n)}\right\|^{2}+\frac{\omega}{S^{2}}\left\|\mathbf{x}-\boldsymbol{\mu}_{s}^{(n)}\right\|^{2}\right),
$$

where $\mathbf{x}$ is the spatial coordinate; $\boldsymbol{\Psi}(\mathbf{x})$ is the corresponding phase retardation feature; and $\boldsymbol{\mu}_{s}^{(n)}$ and $\boldsymbol{\mu}_{p}^{(n)}$ are the prototype (mean) of spatial coordinates and phase retardation features for atom $\mathcal{A}_{n}$, respectively. Each term is normalized by the square of its upper bound. $\omega$ is a balance parameter to adjust the weight between the phase and spatial proximities. In this paper, the dissimilarities of phase retardation features and spatial positions are treated equally; i.e., $\omega$ is set to be 1 for all experiments.

This minimization problem can be solved by associating each pixel to its nearest seed iteratively, and we detail the linear iterative clustering algorithm in Alg. 1 (See Appendix A.1.). Fig. 1 shows an example of the atom generation based on phase retardation features, compared to superpixels based on image intensities. The phase-homogeneous atoms preserve local structure of cell images (Fig. 1.2), i.e., cell regions and halos are segmented into separated atoms, which is consistent with biophysical content of cell images. On the other hand, the intensity-homogeneous atoms are more likely to produce over-segmented noisy clips (Fig. 1.3), because cel1 intensities within cells often vary among subcellular structures.

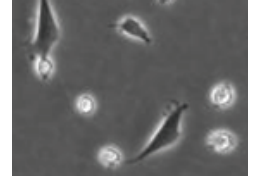

(1.1)

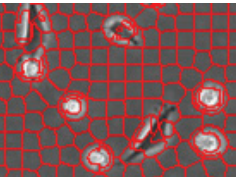

(1.2)

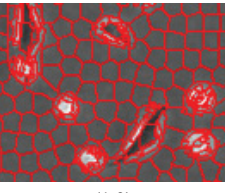

(1.3)
Fig. 1: Atom generation. (1.1) Original phase contrast microscopy image; (1.2) Phase-homogeneous atoms; (1.3) Intensity-homogeneous atoms.

\subsection{Cell segmentation via multi-class spectral analysis}

Cell segmentation is performed by clustering the phasehomogeneous atoms into several clusters, which are unspecified but expected to be interpretable, e.g., bright and dark cells, halos, background, etc.

In revealing the identity of an atom, its neighborhood information plays an important role in addition to the characteristics of itself. For example, without neighboring information, halos and bright cells may not be distinguishable from each other based on phase retardation features within an atom as shown in Fig. 2.2, where the first three principle elements of phase retardation feature vectors are mapped to R, G, and B values of a color image. Specifically, both halos and bright cells are represented by similar distributions of phase retardations, where the dominant one is marked as red color in the figure. In such a case, neighborhood information helps to distinguish them since most halos are around dark cell regions (marked as green color) while bright cells are surrounded by background (marked as black color).

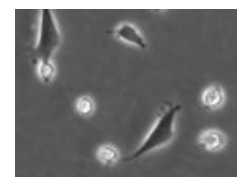

(2.1)

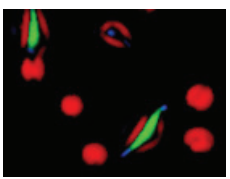

(2.2)

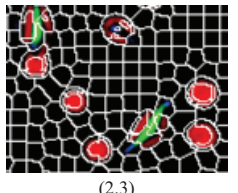

(2.3)
Fig. 2: Color-coded image and atom generation. (2.1) Original phase contrast microscopy image; (2.2) Color-coded phase retardation features corresponding to the original image; (2.3) Phase-homogeneous atoms.

To incorporate the neighborhood information of an atom, we first model the distribution of feature vectors within an atom using a multivariate Gaussian distribution, and then measure the pairwise dissimilarity between neighboring atoms using Jensen-Shannon (JS) divergence [6] of the two Gaussian distributions. Note that the JS divergence is zero if distributions of the corresponding atoms are identical, while approaching positive infinity as they get dissimilar.

Based on the dissimilarity measure, we define neighborhood feature $\tau^{(n)}$ of $\mathcal{A}_{n}$ as the mean of the features in its most distinctive neighboring atom, i.e.,

$$
\boldsymbol{\tau}^{(n)}=\boldsymbol{\mu}^{\left(m^{*}\right)} \text { s.t. } m^{*}=\underset{m: \mathcal{A}_{m} \in \mathcal{N}\left(\mathcal{A}_{n}\right)}{\arg \max } J S D\left(p^{(n)} \| p^{(m)}\right),
$$

where $p^{(n)}$ and $p^{(m)}$ are the multivariate Gaussian distributions of phase retardation features in atom $\mathcal{A}_{n}$ and $\mathcal{A}_{m}$, respectively; $\mathcal{N}\left(\mathcal{A}_{n}\right)$ is the set of $\mathcal{A}_{n}$ 's adjacent atoms, and 
$\boldsymbol{\mu}^{\left(m^{*}\right)}$ is mean vector of phase retardation features in $\mathcal{A}_{m^{*}}$. By concatenating the features within an atom and the features from its neighborhood together, we obtain the descriptor $\mathbf{f}_{n}$ for an atom $\mathcal{A}_{n}$, i.e., $\mathbf{f}_{n}=\left[\boldsymbol{\mu}^{(n)} ; \boldsymbol{\tau}^{(n)}\right]$.

We define the likelihood that a pair of atoms $\mathcal{A}_{n}$ and $\mathcal{A}_{m}$ belong to the same category as

$$
w_{m n}=\exp \left(-\left\|\mathbf{f}_{n}-\mathbf{f}_{m}\right\|^{2}\right) \in(0,1] .
$$

By collecting all $w_{m n}$ together, we construct an affinity matrix $\left[w_{m n}\right]$, and cluster the atoms into $L$ clusters by solving the following combinatorial Dirichlet problem [7]

$$
Y^{*}=\underset{Y}{\arg \max } \frac{1}{L} \sum_{l=1}^{L} \frac{Y_{l}^{T} W Y_{l}}{Y_{l}^{T} D Y_{l}}, \text { s.t. }\left\{\begin{array}{l}
Y \in\{0,1\}^{N \times L} \\
Y \mathbf{1}_{L}=\mathbf{1}_{N}
\end{array},\right.
$$

where $Y_{l}$ is the $l_{\text {th }}$ column of $Y, D$ is a diagonal matrix with $D_{m m}=\Sigma_{i} W_{m i}$, and $\mathbf{1}_{L}$ and $\mathbf{1}_{N}$ in the second constraint denote $L \times 1$ and $N \times 1$ column vectors with all elements being 1 , respectively. Note that the second constraint ensures that each row of $Y^{*}$ contains only one 1 . The $n_{\text {th }}$ row of $Y^{*}$ is the binary cluster indicator of atom $\mathcal{A}_{n}$, i.e., $Y_{n l}^{*}=1$ means that $\mathcal{A}_{n}$ is determined to belong to the $l_{\text {th }}$ cluster.

In order to set the number of clusters $L$, we present a method following the rate distortion theory [6]. Note that if $Y$ is relaxed to take real values, the problem in Eq. (8) reaches its relaxed peak value

$$
\kappa_{L}=\max _{\mathrm{Y}}\left\{\frac{1}{L} \sum_{l=1}^{L} \frac{Y_{l}^{T} W Y_{l}}{Y_{l}^{T} D Y_{l}}\right\}=\frac{1}{L} \sum_{l=1}^{L} \lambda_{l},
$$

where $\lambda_{l}$ is the $l_{\text {th }}$ largest eigenvalue of $D^{-\frac{1}{2}} W D^{-\frac{1}{2}}$. The rate distortion of $\kappa_{L}$ is defined as

$$
d_{r}(L)=\kappa_{L}^{-K},
$$

where $K$ is the number of selected bases in Eq. (1). By increasing the number of clusters $L$ from 2 to a large number (e.g., 100), we obtain a rate distortion curve ( $d_{r}$ vs $\left.L\right)$, as shown in Fig. 3. We utilize a two-segment line to fit the curve, and the best cluster number $L^{*}$ is determined as the intersection of the two line segments

$$
L^{*}=\underset{L}{\arg \min } \frac{L-1}{L_{\max }-1} F E_{1}+\frac{L_{\max }-L}{L_{\max }-1} F E_{2},
$$

where $F E_{1}$ and $F E_{2}$ are fitting errors of the first and second line segments, respectively.

Once the cluster number $L$ in Eq. (8) is determined, we calculate the first $L$ largest eigenvalues of $D^{-\frac{1}{2}} W D^{-\frac{1}{2}}$, and a relaxed continuous solution is given by the corresponding eigenvectors; the clustering result is obtained by finding a discrete solution that is the closest to the optimum and satisfies the binary constraint. The atom clustering procedure is detailed in Alg. 2 (See Appendix A.2.). Finally, cell segmentation is conducted by grouping the neighboring atoms with the same labels.

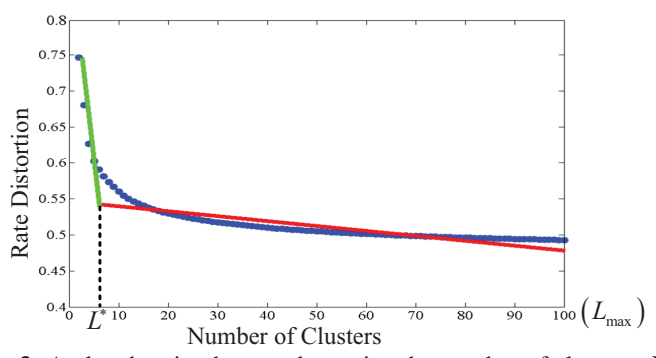

Fig. 3: A plot showing how to determine the number of clusters, $L^{*}$.

\section{EXPERIMENTAL RESULT AND DISCUSSION}

Data: The proposed approach was tested on two sequences of images of two different cell types. Seq. 1 includes images of bovine aortic endothelial cells with resolution $1040 \times 1392$ pixels, and we manually labeled 7020 cells (including 193 mitotic cells) and $1.2 \times 10^{6}$ cell atoms as ground truth. Seq. 2 contains images of muscle stem cells with resolution $696 \times 520$ pixels, and we labeled 1459 cells (including 396 mitotic cells) and $2.6 \times 10^{5}$ cell atoms.

Segmentation and clustering: Sample results of cell segmentation and clustering are illustrated in Fig. 4. In Fig. 4(c), the phase-homogeneous atoms are clustered into different categories: (1) dark cells (i.e., intermitotic cells, marked as orange), (2) bright cells (i.e., mitotic cells, marked as yellow), (3) halos and their adjacent atoms including background, etc. Fig. 4(d) shows segmentation results where cells are marked by red contours.

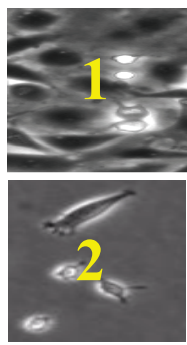

(a)

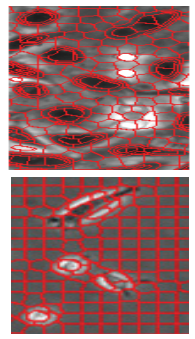

(b)

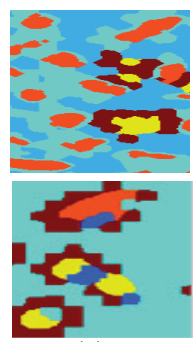

(c)

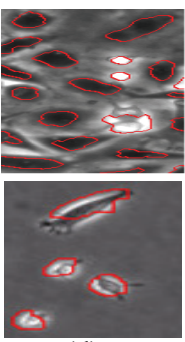

(d)
Fig. 4: Sample results of cell segmentation and clustering. (a) Zoom-in details of input images. (b) Phase-homogeneous atoms. (c) Results of spectral clustering, where clusters are indicated by different colors. (d) Segmentation results marked by red contours.

Comparison and evaluation: For comparison, we implemented two other cell segmentation approaches: (1) partition images into intensity-homogeneous atoms, and utilize spectral clustering for segmentation (Intensity-Atom), and (2) cluster pixels based on pixelwise phase retardation features without the atom segmentation step (Phase-Pixel). Sample results illustrated in Fig. 5 clearly show that our proposed algorithm produces quality cell segmentation results (Fig. 5(b)). On the other hand, segmentation of IntensityAtom often results in fuzzy segmentation on the boundary and over-segmented clips (Fig. 5(c)), because pixel intensity 
within a cell is sometimes similar to that of the background, and subcellular structures within a cell exhibit dissimilar appearances. The results of Phase-Pixel are not comparable to our methods either (Fig. 5(d)) due to the noise in both cell regions and the background. Furthermore, Phase-Pixel is time costly due to the huge number of instances, since the computational complexity of spectral clustering is $\mathcal{O}\left(N^{3}\right)$ where $N$ is the number of instances.
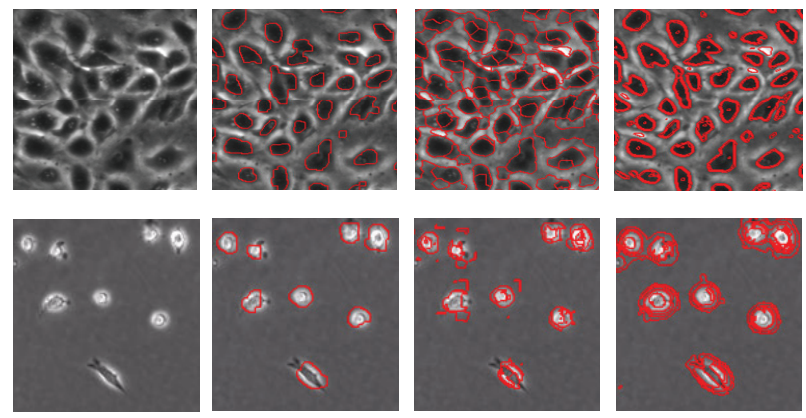

(b)
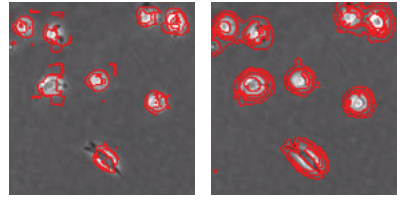

(a)

(c)

(d)

Fig. 5: Comparison among different segmentation methods. The top and bottom rows show results on Seq. 1 and Seq. 2, respectively. (a) Zoom-in details of input images, (b) Results of our method, (c) Results of IntensityAtom, (d) Results of Phase-Pixel.

We quantitatively evaluate the segmentation performance using the normalized probabilistic rand index (NPR) [8], which penalizes both cases of over- and under-segmentations. The quantitative comparison is reported in Table 1, which demonstrates that our proposed method outperforms the other two methods. Intensity-Atom and Phase-Atom show relatively low NPR indices as over- and under-segmentations occur more frequently, caused by fuzzy segmentation and noisy segmented clips.

Table 1: NPR Index on Cell Segmentation

\begin{tabular}{|c|c|c|c|c|c|}
\hline \multicolumn{3}{|c|}{ Seq.1 } & \multicolumn{3}{c|}{ Seq.2 } \\
\hline $\begin{array}{c}\text { Phase- } \\
\text { Atom }\end{array}$ & $\begin{array}{c}\text { Intensity- } \\
\text { Atom }\end{array}$ & $\begin{array}{c}\text { Phase- } \\
\text { Pixel }\end{array}$ & $\begin{array}{c}\text { Phase- } \\
\text { Atom }\end{array}$ & $\begin{array}{c}\text { Intensity- } \\
\text { Atom }\end{array}$ & $\begin{array}{c}\text { Phase- } \\
\text { Pixel }\end{array}$ \\
\hline 0.87 & 0.42 & 0.76 & 0.85 & 0.72 & 0.69 \\
\hline
\end{tabular}

Since our segmentation method is based on clustering among atoms, cells with different properties are separately clustered. This additional information is useful for cell stage classification because cells undergoing the same stage (e.g., mitosis) are more likely to be clustered into the same cluster. To quantitatively validate this argument, we manually labeled mitotic cells and examined whether they are indeed grouped into the same cluster. As expected, $96.6 \%$ of mitosis in Seq. 1 and $93.9 \%$ of mitosis in Seq. 2 were clustered to the same cluster, and only $7.7 \%$ and $6.1 \%$ of the elements in the cluster were non-mitotic cells in Seq. 1 and Seq. 2, respectively. This result shows that our proposed algorithm can provide quality candidates for cell stage classification or event detection methods, e.g., [9].

\section{CONCLUSION}

In this paper, we propose a cell segmentation algorithm via spectral analysis over phase retardation features derived in [4]. Based on the similarity between phase retardation features, a phase contrast microscopy image is first partitioned into phase-homogeneous atoms. Cell segmentation is then performed by a multi-class spectral clustering technique based on the features from each atom and its neighboring atoms. Experimental results compared with manual annotation and against alternative methods show our method's effectiveness on cell segmentation and its potential for cell event detection or stage classification.

\section{REFERENCES}

[1] D. Murphy, Fundamentals of Light Microscopy and Electronic Imaging, chapter Phase Contrast Microscopy and Dark-Field Micorscopy, pp. 97-116, Wiley, 2001.

[2] N. N. Kachouie, P. Fieguth, and E. Jervis, "Watershed deconvolution for cell segmentation," in Proc. International Conference on Engineering in Medicine and Biology Society, pp. 375-378, 2008.

[3] D. Padfield, J. Rittscher, N. Thomas, and B. Roysam, "Spatio-temporal cell cycle phase analysis using level set$\mathrm{s}$ and fast marching methods," Medical Image Analysis, vol. 13, no. 1, pp. 143 - 155, 2009.

[4] H. Su, Z. Yin, T. Kanade, and S. Huh, "Phase contrast image restoration via dictionary representation of diffraction patterns," in Proc. International Conference on Medical Image Computing and Computer Assisted Intervention, pp. 615-622, 2012.

[5] Z. Yin, T. Kanade, and M. Chen, "Understanding the phase contrast optics to restore artifact-free microscopy images for segmentation," Medical Image Analysis, vol. 16, no. 5, pp. $1047-1062,2012$.

[6] T. M. Cover and J. A. Thomas, Elements of information theory, Wiley-Interscience, New York, NY, USA, 1991.

[7] S. X. Yu and J. Shi, "Multiclass spectral clustering," in Proc. International Conference on Computer Vision, pp. 313-319, 2003.

[8] R. Unnikrishnan, C. Pantofaru, and M. Hebert, "Toward objective evaluation of image segmentation algorithms," IEEE Trans. on Pattern Analysis and Machine Intelligence, vol. 29, no. 6, pp. 929 -944, 2007.

[9] S. Huh, D. F. E. Ker, R. Bise, M. Chen, and T. Kanade, "Automated mitosis detection of stem cell populations in phasecontrast microscopy images," IEEE Trans. on Medical Imaging, vol. 30, no. 3, pp. 586-596, 2011. 


\section{A. APPENDIX}

\section{A.1. Algorithm for atom generation}

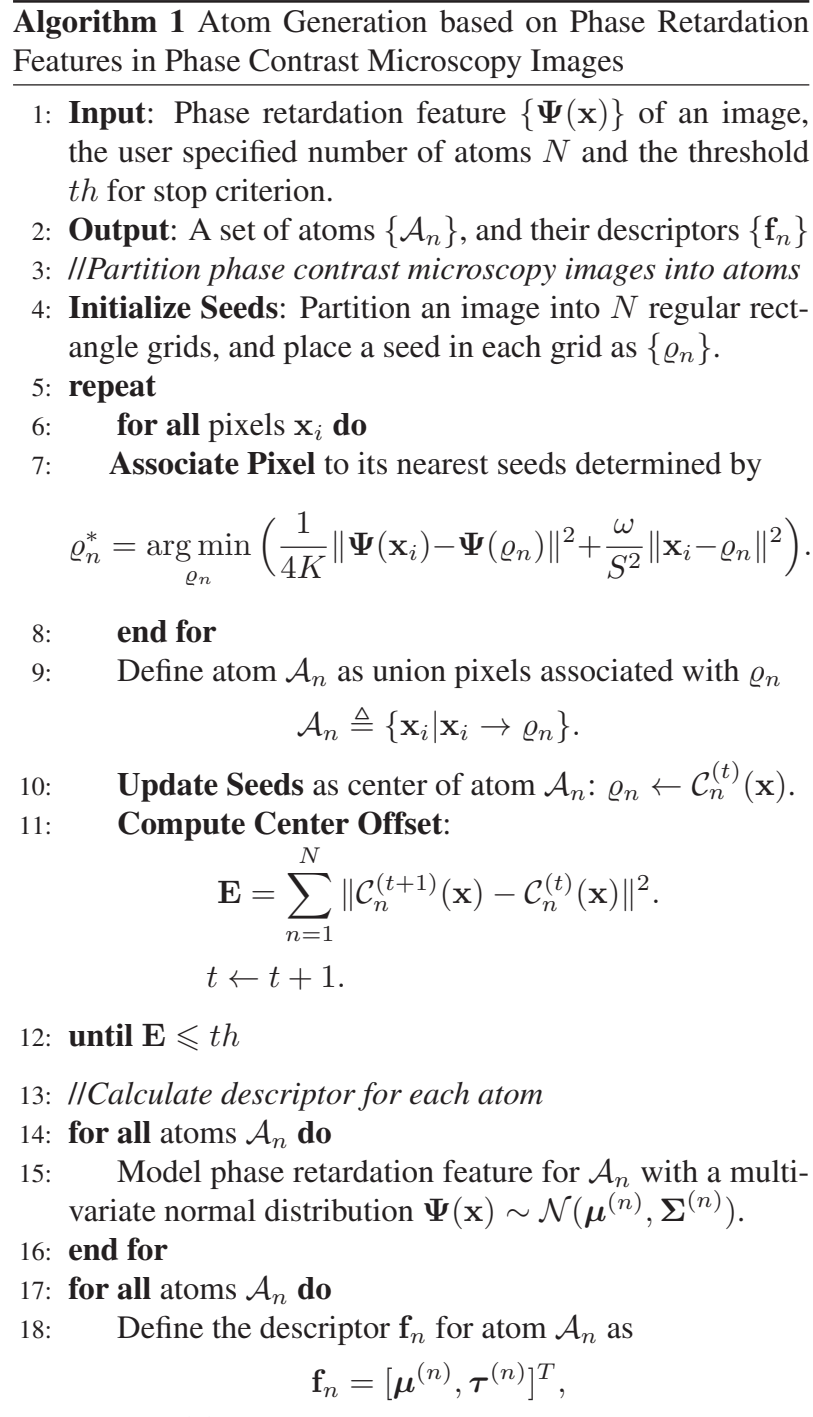

where $\boldsymbol{\mu}^{(n)}$ is the mean phase retardation feature vector of atom $\mathcal{A}_{n}$, and $\tau^{(n)}$ is its neighboring characteristic

$$
\boldsymbol{\tau}^{(n)}=\boldsymbol{\mu}^{\left(m^{*}\right)} \text { s.t. } m^{*}=\underset{m: \mathcal{A}_{m} \in \mathcal{N}\left(\mathcal{A}_{n}\right)}{\arg \max } J S D\left(p^{(n)} \| p^{(m)}\right) .
$$

19: end for

\section{A.2. Algorithm for cell segmentation}

Algorithm 2 Cell Segmentation based on Multi-class Spectral Clustering

1: Input: Atoms $\left\{\mathcal{A}_{n}\right\}$ of an image, and their descriptors $\left\{\mathbf{f}_{n}\right\}$.

2: Output: Cell segmentation result $Y^{*}$

3: //Construct the affinity matrix

4: Compute similarity between atoms $\mathcal{A}_{n}$ and $\mathcal{A}_{m}$

$$
w_{m n}=\exp \left(-\left\|\mathbf{f}_{n}-\mathbf{f}_{m}\right\|^{2}\right) \in(0,1],
$$

and construct the affinity matrix as $W=\left[w_{m n}\right]$.

5: Compute the diagonal degree matrix $D$ as

$$
D=\operatorname{Diag}\left(\left[\sum_{i=1}^{N} w_{1 i}, \ldots, \sum_{i=1}^{N} w_{N i}\right]\right) .
$$

6: //Formulate the optimization problem

7: Formulate cell segmentation as a combinatorial Dirichlet minimization problem as

$$
Y^{*}=\underset{Y}{\arg \max } \frac{1}{L} \sum_{l=1}^{L} \frac{Y_{l}^{T} W Y_{l}}{Y_{l}^{T} D Y_{l}} \text {, s.t. }\left\{\begin{array}{l}
Y \in\{0,1\}^{N \times L} \\
Y \mathbf{1}_{L}=\mathbf{1}_{N}
\end{array},\right.
$$

where $Y^{*}$ is the atom indicator matrix.

8: //Determine the optimal cluster number

9: Determine the cluster number $L^{*}$ as

$$
L^{*}=\underset{L}{\arg \min } \frac{L-1}{L_{\max }-1} F E_{1}+\frac{L_{\max }-L}{L_{\max }-1} F E_{2},
$$

where $F E_{1}$ and $F E_{2}$ are the fitting errors of line segments for the rate distortion defined in Eq. (10), respectively.

10: //Solve the optimization problem

11: Calculate the eigenvectors of $D^{-\frac{1}{2}} W D^{-\frac{1}{2}}$ that correspond to its first $L^{*}$ largest eigenvalues

$$
V=\left[\mathbf{v}_{1}, \cdots, \mathbf{v}_{L^{*}}\right] .
$$

12: Find the optimal solution in Eq. (8) by

$$
Y^{*}=D^{-\frac{1}{2}} V=D^{-\frac{1}{2}}\left[\mathbf{v}_{1}, \cdots, \mathbf{v}_{L^{*}}\right] .
$$

13: //Cell segmentation and stage classification

14: Find the optimal discrete solution via an iterative refining procedure in [7], and cell segmentation is thus realized by grouping the neighboring atoms with the same labels. 


\section{A.3. Supplemental figures}

- Flowchart of our proposed cell segmentation and stage classification algorithm

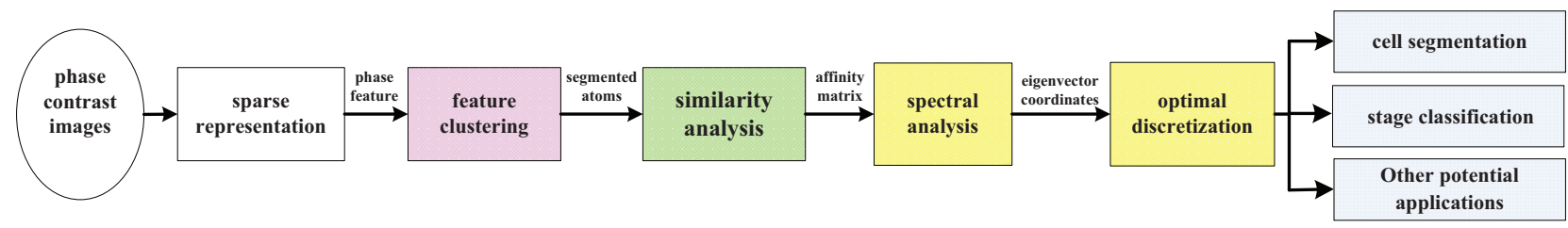

Fig. 6: Steps of our proposed cell segmentation algorithm. Phase contrast microscopy images are restored with sparse representation algorithm [4], and partitioned into phase-homogeneous atoms by clustering neighboring pixels with similar phase retardation features. After modeling the distribution of phase retardation feature with a multivariate Gaussian model, we utilize the Jensen-Shannon divergence [6] to measure the dissimilarity between an atom and its neighborhood. An affinity matrix is then constructed to characterize the similarity between any pair of atoms. Furthermore, we employ multi-class spectral clustering technique to classify the atoms into several clusters, e.g., background, intermitotic cells, mitosis cells, etc.

- Sample results for Seq. 1 (Detailed results of Fig. 4)

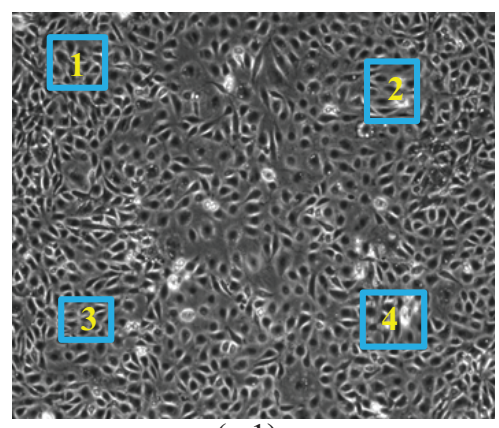

(a.1)

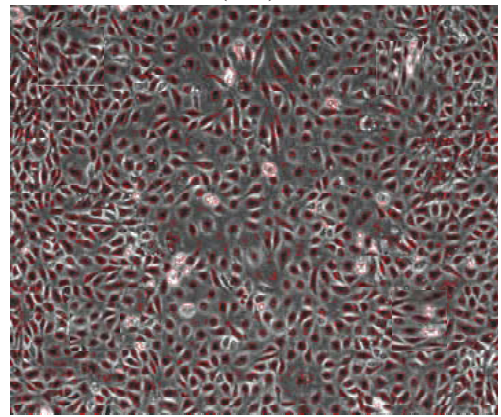

(a.2)
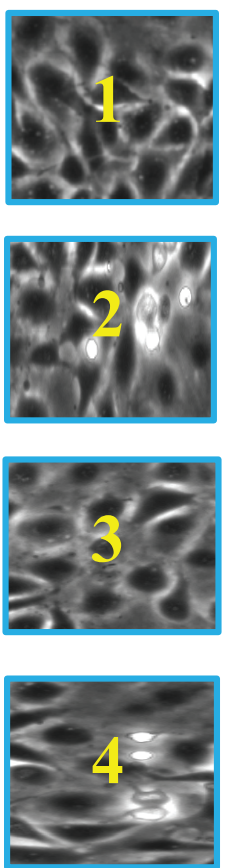

(b)
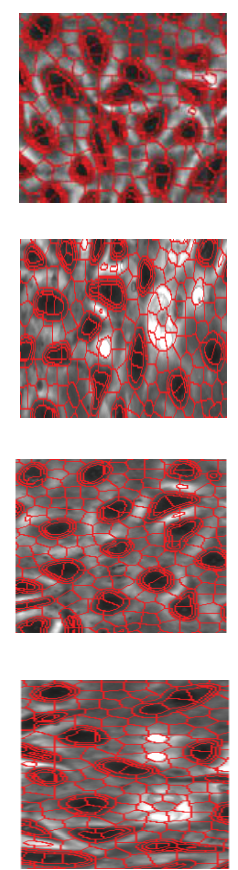

(c)
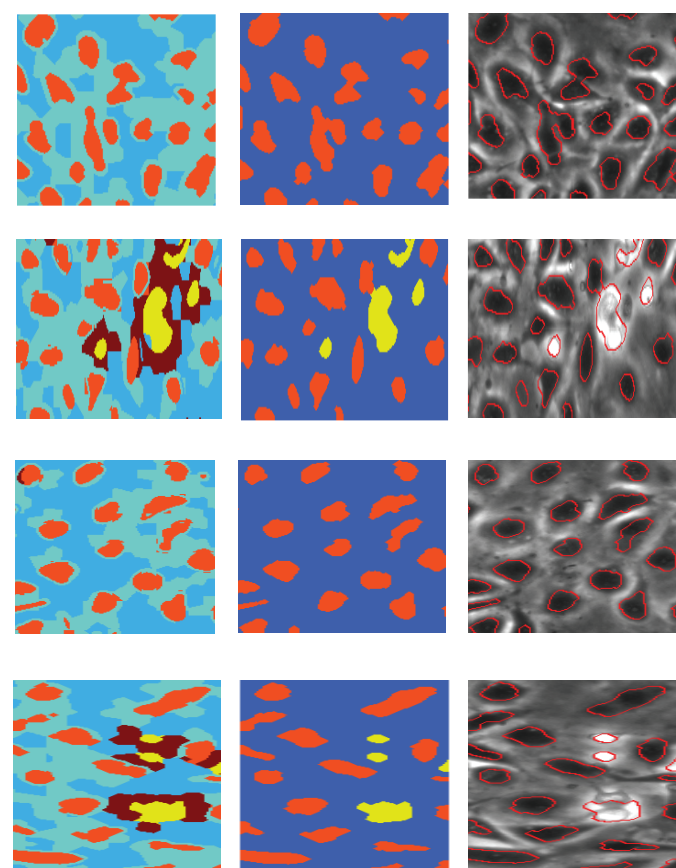

(d)

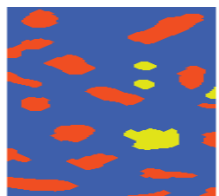

(e)

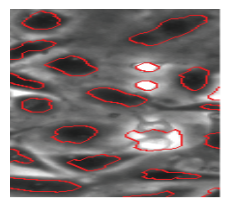

(f)

Fig. 7: Sample results of cell segmentation and stage classification for Seq. 1. (a.1) Phase contrast microscopy image. (a.2) Cell segmentation result on the image. (b) Zoom-in details of input phase contrast microscopy image. (c) Results of phase-homogeneous atoms, which show that the atoms preserve local structures of cell images, i.e., cell regions and halos are segmented into separated atoms. (d) Results of spectral clustering where each cluster is indicated by a different color. The atoms are categorized into dark cells, bright cells, halos and their adjacent atoms including background, etc. (e) Results of cell classification and segmentation, where bright cells (mitosis cells) and dark cells (intermitotic cells) are separately clustered. (f) Segmentation results where cells are marked by red contours. 
- Sample results for Seq. 2 (Detailed results of Fig. 4)

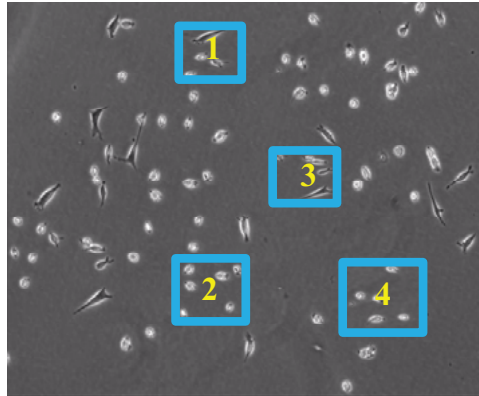

(a.1)

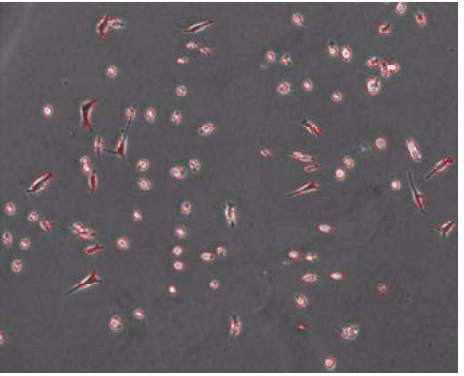

(a.2)
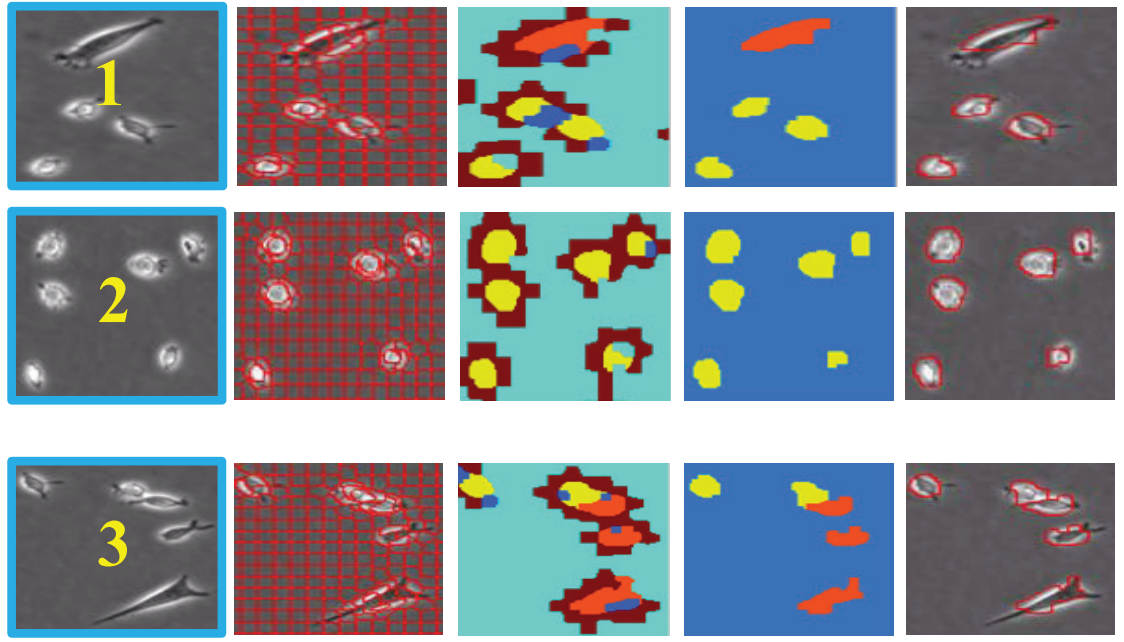

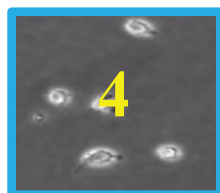

(b)

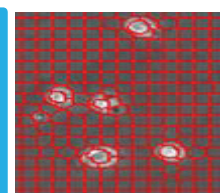

(c)

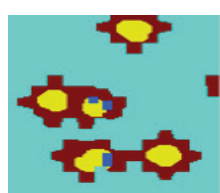

(d)

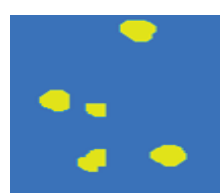

(e)

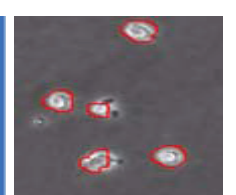

(f)

Fig. 8: Sample results of cell segmentation for Seq. 2. (a.1) Phase contrast microscopy image. (a.2) Cell segmentation result on the image. (b) Zoom-in details of input phase contrast microscopy image. (c) Results of phase-homogeneous atoms, which show that the atoms preserve local structures of cell images, i.e., cell regions and halos are segmented into separated atoms, which consists with biophysical content of cell images. (d) Results of spectral clustering, where each cluster is indicated by a different color. The atoms are categorized into dark cells, bright cells, halos and their adjacent atoms including background, etc. (e) Results of cell classification and segmentation, where bright cells (mitosis cells) and dark cells (intermitotic cells) are separately clustered. (f) Segmentation results where cells are marked by red contours.

\section{- Comparison among different segmentation algorithms (Detailed results of Fig. 5)}
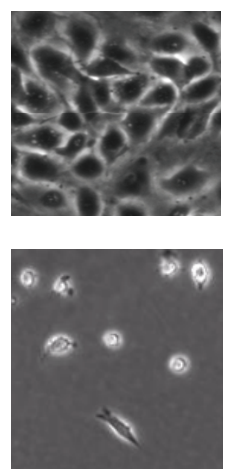

(a)
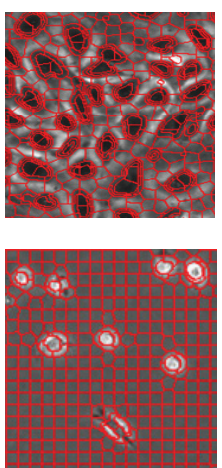

(b)
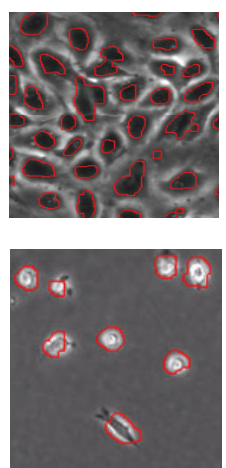

(c)
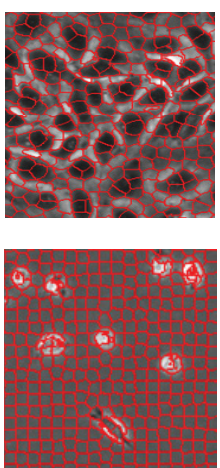

(d)
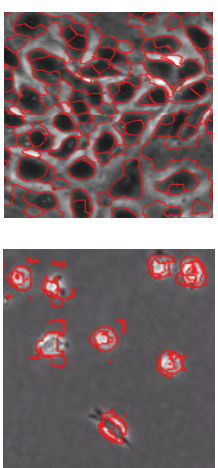

(e)
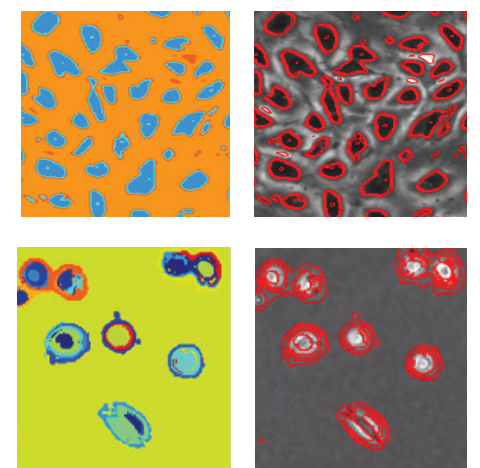

(f)

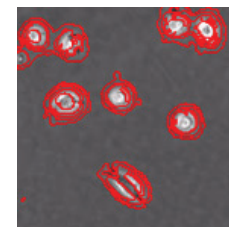

$(\mathrm{g})$

Fig. 9: Comparison of our proposed algorithm with other methods: segmentation based on image intensity (Intensity-Atom) and segmentation based on pixelwise phase retardation feature (Phase-Pixel). The top and bottom rows show results on Seq. 1 and Seq. 2, respectively. (a) Zoom-in details of input phase contrast microscopy images. (b) Results of phase-homogeneous atom generation. (c) Segmentation results where cells are marked by red contours. (d) Results of intensity-homogeneous atoms. (e) Cell segmentation results based on the intensity-homogeneous atoms, which show a fuzzy segmentation between cells and background (top row) due to the low contrast between cells and background, and noisy over-segmented clips (bottom row) due to dissimilarly between subcellular structures. (f) Spectral clustering results based on pixelwise phase retardation features. By Eq. (11), a much larger number of clusters was selected. (g) Segmentation results based on pixelwise clustering, which shows noisy over-segmented clips and fuzzy contours. 